\title{
Recent advances in Wilson disease
}

\author{
Maryam Moini ${ }^{1,2}$, Uyen To ${ }^{3}$, Michael L. Schilsky ${ }^{3}$ \\ ${ }^{1}$ Gastroenterohepatology Research Center, Shiraz University of Medical Sciences, Shiraz, Iran; ${ }^{2}$ Division of Gastroenterology, University of Toronto, \\ Toronto, Canada; ${ }^{3}$ Department of Medicine and Surgery, Division of Digestive Diseases and Transplantation and Immunology, Yale University, New \\ Haven CT, USA \\ Contributions: (I) Conception and design: ML Schilsky; (II) Administrative support: ML Schilsky; (III) Provision of study materials or patients: None; \\ (IV) Collection and assembly of data: All authors; (V) Data analysis and interpretation: None; (VI) Manuscript writing: All authors; (VII) Final \\ approval of manuscript: All authors. \\ Correspondence to: Dr. Michael L. Schilsky. Department of Medicine and Surgery, Division of Digestive Diseases and Transplantation and \\ Immunology, Yale University, New Haven, CT, USA. Email: michael.schilsky@yale.edu.
}

\begin{abstract}
Wilson disease (WD) is rare genetic disorder that presents with varied phenotype that can at times make the diagnosis challenging. Medical treatments are available, but there are still unmet needs for patients. Since life-long therapy is necessary, adherence to medical therapy and best practices for monitoring and individualizing therapy continue to evolve. Studies are ongoing that address some of these issues. In the current review we focused our attention to recent advances in the diagnosis of WD, current medical treatments, future potential therapies and treatment monitoring. We include discussion of new methodology for detection and quantitation of ophthalmologic signs of WD, new brain imaging modalities for early detection of neurologic involvement in patients and potential new diagnostic methodology using blood samples that may be applicable to newborn screening and adult disease diagnosis. In addition, there are new strategies aimed at improving adherence and outcomes with currently available therapies, including once daily chelation dosing and discussion of the efficacy of different zinc salt compounds. With respect to new therapies with different mechanisms of action, we discuss studies on Bis-choline tetrathiomolybdate (TTM) in patients, pre-clinical studies of a novel chelator methanobactin and other animal studies exploring cures for WD with gene therapy using adeno-associated vectors (AAVs) that introduce ATP7B into liver cells. There are also promising advances in the more accurate measurement of non-ceruloplasmin bound copper and exchangeable copper in the circulation which would potentially help with monitoring and individualization of treatment and possibly play a role in future disease diagnosis.
\end{abstract}

Keywords: Wilson disease (WD); D-penicillamine; Trientine; Zinc salts; Bis-choline tetrathiomolybdate (TTM); methanobactin; adeno-associated virus vector; cell therapy

Received: 16 February 2020; Accepted: 19 March 2020; Published: 05 April 2021.

doi: $10.21037 /$ tgh-2020-02

View this article at: http://dx.doi.org/10.21037/tgh-2020-02

\section{Introduction}

Progress over the last century has evolved from the formal recognition of Wilson disease (WD) as a neurologic disorder with associated liver disease (1) to the understanding that WD is an autosomal recessive inherited disorder of copper metabolism (2). Even before the responsible gene for $\mathrm{WD}, A T P 7 B$, was identified (3), it was known that excess copper, in particular in the liver and the central nervous system, underlay the pathophysiology of this disease and its wide array of clinical phenotype. As such, development of medical therapy focused on the removal of copper to arrest disease progression and even reverse some of the clinical manifestations of copper toxicity.

All current medical treatments for WD require lifelong therapy to maintain the health of the patient. As with treatment of any chronic disease, the requirement for daily therapy, sometimes with multiple daily dosages of 
Table 1 Current advances in diagnosis and management of Wilson disease

New modalities for diagnosis

- Anterior segment optical coherence tomography for detection and quantification of Kayser Fleischer ring (5)

- Dynamic PET analysis with copper-64 chloride detecting increased accumulation and reduced clearance of copper from the liver of mice model of WD (6)

- MR spectroscopy of brain for detection of early neurological changes of WD (7)

- Liquid chromatography multiple reaction monitoring mass spectrometry for quantification of ATP7B protein in dried blood as potential screening tool for WD (8)

- Relative exchangeable copper, the ratio of CuEXC to total serum copper $(9,10)$

New therapeutic agents and treatment strategies

Therapeutic agents

- Once daily dosing of trientine for better patient's compliance $(11,12)$

- Bis-choline Tetrathiomolybdate with advantage of neurologic improvement $(13,14)$

- Zinc salts with variable compounds have comparable efficacy in treatment (15)

- Methanobactin with potent affinity for copper showed promising results for prevention of copper induced mitochondrial injury in hepatocytes and histology improvement in animal model of WD (16-18)

Gene therapy

- Adeno-associated vector encoding human ATP7B cDNA in the hepatocytes of the mouse model of WD showed adequate expression of ATP7B with reduce hepatic copper and prevention of hepatic injury (19)

- A shorter vector coding for a miniATP7B protein was shown to be effective in achieving long-term copper hemostasis in mouse model (20)

Cell therapy

- Transplantation of normal hepatocytes was associated with prevention of fulminant hepatitis, decreased inflammation and increased short term survival in rat model of WD $(21,22)$

Monitoring of treatment

- Anion exchange chromatography-triple quadrupole inductively coupled plasma mass spectrometry for simple quantification of ceruloplasmin for a more accurate determination of non-ceruloplasmin copper level (23)

cDNA, complementary DNA; CuEXC, exchangeable copper; MR, magnetic resonance; PET, positron emission tomography; WD, Wilson disease.

medication apart from food, has led to non-adherence with treatment in up to half of patients and recurrent or new disease symptoms or even death from liver failure in some non-adherent patients (4). Furthermore, some patients experience side effects of treatment. We discuss here the current advances in diagnostic tools, management and monitoring for WD with summarization of key points in Table 1.

\section{Diagnosis}

The diagnosis of WD has been historically based on clinical history and biochemical evaluation. The clinical findings of Kayser Fleischer (KF) rings, cirrhosis, neurological or psychiatric complications in various combinations can be used to diagnose individuals with WD. However, it can be difficult when patients present with only one or two of the clinical findings. Biochemical evaluation with serum copper, serum ceruloplasmin, liver enzymes, urine copper excretion, hepatic copper quantitation and (if available) genetic testing are also helpful in establishing the diagnosis, but each has its' limitations. Imaging, such as MRI of the brain, can be useful if abnormalities are seen in the basal ganglia. As early diagnosis is essential to prevent long term complications, several advances in diagnostic imaging and genetic testing have proved helpful in diagnosing WD (24). 
Recent advances in evaluating for KF rings were helpful in both identifying and quantifying the rings. KF rings are usually identified by ophthalmologists via a slit lamp biomicroscopy but can be difficult to quantify. In anterior segment optical coherence tomography, the grey scale detects the KF rings by its' hyper reflective band at the level of the Descemet's membrane in the cornea. On a color scale the rings can be seen as a yellow orange band. The anterior segment optical coherence tomography has been proposed as a sensitive method for detection of KF rings by a nonophthalmologist and also for quantification of the KF rings that can be followed over time for patients undergoing treatment (5).

Imaging advances such as positron emission tomography (PET) imaging and magnetic resonance spectroscopy have also helped in diagnosis, by improving our understanding copper metabolism and by potential early detection of neurological abnormalities. Dynamic PET analysis using copper-64 chloride as a tracer was shown in ATP7B knock out mice to detect increased accumulation and reduced clearance of copper from the liver (6). It also showed copper was retained in other organs such as kidney, heart and lungs. These studies demonstrated the potential not only for diagnostic use but also for monitoring copper accumulation in the body. Other advances in imaging include use of magnetic resonance (MR) spectroscopy of tissues in the pediatric population. In a case controlled prospective study, 26 pediatric patients with WD were compared to 26 healthy control (7). The levels of N-acetyl aspartate (NAA), choline (Cho), and creatine $(\mathrm{Cr})$, as well as the metabolite ratios of NAA/Cho, NAA/Cr, and Cho/ $\mathrm{Cr}$ in brain tissue as determined by MR spectroscopy was significantly decreased in WD patients compared to healthy individuals. A more severe reduction in NAA, NAA/Cr, and NAA/Cho was observed in WD patients with mixed neurologic and hepatic involvement compared with those with liver only involvement. This potentially can serve as a method for detecting early neurological involvement in pediatric patients with WD.

Unfortunately, despite the availability of clinical, biochemical and genetic testing, many patients come with a delayed diagnosis of WD. Currently there are no reliable biomarkers or screening tests in pediatric or neonate patients, as ceruloplasmin has been studied in this population with inconsistent circulating levels of the protein due to age and other potential confounding factors. One method that has been recently discussed as a potential diagnostic tool is liquid chromatography multiple reaction monitoring mass spectrometry (8). This technology allows for potential identification and quantification of proteins that may not be present in large amounts in dried blood spots. Preliminary findings show that ATP7B may be decreased in blood spot specimens from patients with WD compared to non-affected individuals (8), however further studies are needed for validation of the methodology.

\section{Management of WD}

The mainstay of current management for WD is based on inducing a negative copper body balance through decreased intake from diet, blocking absorption, or by increasing copper excretion.

Chelating agents are a class of drugs whose mechanism of action is promotion of the excretion of excess body copper through the urine, or in the case of tetrathiomolybdate (TTM), into bile. British anti-Lewisite (BAL) or 2,3-dimercaptopropanol was the first chelating agent introduced as an effective treatment for WD in 1951 which was administered through intramuscular injection $(25,26)$. D-penicillamine was the first oral chelating agent used for treatment of WD (27), and this was followed by the development of trientine (diethylene tetramine) that is approved for patients intolerant of $\mathrm{d}$-penicillamine. These chelating agents work by binding with plasma copper and excreting in urine. D-penicillamine may also induce release of intracellular stores of metallothionein, an endogenous copper chelator $(28,29)$. D-penicillamine, however, is associated with many side effects causing a relatively high rate of intolerance (over 30\%) over time. The main concern with D-penicillamine therapy is the risk of deterioration of neurologic manifestations during treatment initiation in patients with neurologic manifestations of their WD $(30,31)$. Trientine may have fewer side effects (32), however about $20 \%$ of patients initially treated with this medication can also develop paradoxical neurologic worsening.

Zinc salts as a treatment for WD acts by creating a negative copper balance by decreasing absorption of dietary copper from gut. Zinc induces metallothionein in enterocytes that binds the ingested copper and traps it within the cells. The enterocytes are then excreted into the stool (24). Zinc salts have gained popularity for treatment of very young children with WD, treatment of asymptomatic WD patients, and treatment of symptomatic patients who experienced adverse effects with chelation therapy. Limitations of zinc treatment include gastrointestinal upset (leading to intolerance in about one third of patients) 
and decreased adherence due to multiple daily doses administered away from meals.

\section{New therapeutic agents and new treatment strategies}

\section{Trientine}

Trientine appears to have less side effects and superior tolerability compared with D-penicillamine, and is becoming a preferred first line treatment for WD. The recommended dose is $20 \mathrm{mg} / \mathrm{kg}$ initially and later $\sim 15 \mathrm{mg} / \mathrm{kg}$ given as divided doses 2-4 times daily. Poor compliance with medication use is often a significant barrier for optimized management of WD (33). The possible efficacy of once daily dose of trientine as a maintenance therapy for WD was suggested by case studies and in a limited pilot study carried out on 8 WD patients for 12 months $(11,12)$. The once daily dosing of trientine was felt by these patients to improve their adherence to treatment and was more convenient given the necessity to take the medication apart from food. This study demonstrated the stability of their biochemical status and good copper excretion on the once daily trientine. Larger trials with longer duration of follow up and testing of dose response for treatment are required to address the safety, long-term efficacy and cost effectiveness of single dose trientine treatment for maintenance therapy in WD.

\section{TTM}

TTM was studied as initial treatment for WD patient with neurologic symptoms (34-36). TTM is a potent decoppering agent with multiple mechanisms of action; it interferes with copper absorption from the gut when given with food, it binds plasma copper in a tripartite complex with albumin, and also has a role for copper removal from metallothioneins in tissues, including hepatocytes with the resultant increase in biliary excretion of copper (24). There is also a proposed role of anti-angiogenesis, antiinflammatory and anti-fibrosis for TTM through decoppering and cytokine inhibition (37). The ammonium salt of TTM used initially for treatment of WD was not stable and was therefore difficult to store or use clinically (38). Current clinical trials are using a more stable TTM compound, Bis-choline TTM, for the treatment of WD. Phase I and II studies are complete and phase III is ongoing. The results from phase II were promising with favorable safety profile overall. Though up to $1 / 3$ had elevations in transaminases early after TTM initiation, these resolved with dose reduction or discontinuation and were not associated with concomitant elevation of bilirubin. On further treatment with TTM during extension beyond the initial 6 months, stable liver function was maintained, even in those with cirrhosis. The patient also demonstrated a reduction of non-ceruloplasmin bound serum copper to within normal ranges between 8-12 weeks after initiation of treatment, and most had improvement of neurologic symptoms $(13,14)$. The current phase III study is recruiting WD patients naïve to treatment and those on maintenance therapy for their WD (NCT03403205).

\section{Zinc salts}

Zinc salts are an alternative treatment for patients intolerant of chelation agents and also for initial and maintenance therapy of asymptomatic or stable patients. Initial studies in the US utilized zinc acetate as the favored zinc salt, and there was a perception that alternative zinc agents might not be as well absorbed or as effective. In order to address these questions, Camarata et al. retrospectively reviewed a single center experience of patients who were on zinc therapy for their WD with zinc acetate and also with alternative zinc salts (15). The absorption of the zinc was found to be good with zinc acetate but also with other zinc salts, including zinc gluconate and zinc picolinate. There was a similar efficacy with respect to achieving a normal ALT and urine copper excretion of $<100 \mathrm{mcg}$ per day for the different zinc salts. Whether those with normal ALT and appropriate copper excretion on zinc treatment are absolutely prevented from disease progression of their WD over time remains to be determined, however the majority of those achieving both of these parameters in this cohort were clinically stable and doing well.

\section{Metbanobactin}

Methanobactin is a peptide produced by a bacterium named methylosinus trichosporium with a potent affinity for copper. Methanobactin within hepatocytes remove toxic copper from cells and prevented mitochondrial injury. Treatment with methanobactin reversed mitochondrial dysfunction and liver injury in animal models of WD in the acute phase of copper toxicity (16). A short course of treatment with methanobactin was shown to effectively remove the excess liver copper content in WD disease 
rat model, an effect that was sustained for several weeks after stopping treatment (17). High copper affinity of methanobactin and its rapid action for copper removal from hepatocytes in animal models suggests its possible application for treatment of acute cases of WD. More studies and clinical trials are required.

In a second study using this same animal model, animals were fed a high fat diet that compounded the injury of copper to the liver. Treatment with methanobactin prevented the mitochondrial injury and improved liver histology. Interestingly, this study highlighted the convergence of pathways for liver injury for steatohepatitis and for WD with the potential synchronous oxidative injury to the hepatocytes (18). This likely has relevance for patients with WD who may have exacerbation of their injury if they have concomitant NASH, or have progression of their liver disease despite treatment that is directed against the copper toxicity alone.

\section{Gene therapy}

Gene replacement therapy theoretically can provide a potential cure for genetic disorders. WD as a monogenic inherited disorder with impaired ATP7B function, could possibly be treated with gene therapy if sufficient transgene expression of $A T P 7 B$ in hepatocytes could be maintained over time. Lentiviral gene transfer that integrates the $A T P 7 B$ gene into the genome has been shown to be effective for ameliorating disease progression in animal models of WD (39). However, there are concerns that using such integrative techniques could cause oncogenesis. Adeno-associated virus is an alternative vector for gene therapy that is gaining popularity due to its ability for direct extra-chromosomal gene transfer to hepatocytes. Using an adeno-associated vector (AAV) encoding human $A T P 7 B$ complementary DNA (cDNA) in the hepatocytes of the Atp $7 b^{-/-}$WD mouse, adequate expression of ATP7B was achieved to reduce hepatic copper and prevent liver injury (19). However, the large size of $A T P 7 B$ cDNA affected the vector's cloning capacity for production of efficient gene transfer. To overcome this limitation, a shorter vector coding for a miniATP7B protein was generated that was effective in achieving long-term copper hemostasis in this mouse model of WD (20). Based on these studies, AAV gene transfer for the genetic correction of WD in humans is being planned. Issues to overcome in AAV directed therapy include the potential for using neutralizing antibodies in some patients that have pre- existent antibodies and patients who develop antibodies after administration of AAV. Alternatives to re-introducing the gene via the same AAV vector as hepatocytes turnover would also help to maintain the therapy by allowing safe gene transfer into previously treated individuals.

\section{Cell therapy}

Phenotype correction of WD is possible through liver transplantation. Transplantation of adequate amount of healthy hepatocytes may restore ATP7B related ATPase activity at the level adequate for a normal copper metabolism. In WD rat model, transplantation of normal hepatocytes has been shown to be associated with prevention of fulminant hepatitis, decreased inflammation and increased short term survival (21). Repeated cycles of cell transplantation may require to achieve adequate clinical improvement (22). There are no reported human trials of cell transplantation with WD. Potential limitations with this approach include the need for transplantation of large amount of cells, the requirement for long term immunosuppression and differences in the success of implantation if there is already disease within the liver that alters the livers' microcirculation.

\section{Monitoring of treatment}

Monitoring of treatment is of significant importance in the management of WD. An optimized therapy plan is achieved when there is evidence for biochemical and clinical improvement with minimal management related adverse events and good patient compliance. Monitoring should include regular clinic visits for physical examination and history for both disease and medication related side effects. Laboratory testing should also be done to evaluate for adequacy of treatment. Frequency of monitoring varies with the stage of disease. More frequent monitoring is required in initiation of therapy and less in the stable phase of disease. Laboratory testing include liver function tests, serum copper, ceruloplasmin, complete blood count, $24 \mathrm{hr}$ urine and for those on chelating agents urinalysis (24). Calculated non-ceruloplasmin bound copper using ceruloplasmin and serum copper measurement can provide the best estimate for adequacy of any type of treatment (chelating or nonchelating therapy) (24). A level between less than $15 \mathrm{mcg} / \mathrm{dL}$ but more than $5 \mathrm{mcg} / \mathrm{dL}$ is considered as an index of treatment efficacy while avoiding copper deficiency. However, this method is subjected to potential errors based 
on the accuracy of ceruloplasmin and copper measurement and thus there have been recent advances into alternative methods of monitoring copper balance.

One method of monitoring and response to therapy is measuring exchangeable copper. Exchangeable copper (CuEXC) assays use specific chelating agent to measure copper "exchanged" or mobilized from proteins and peptides other than ceruloplasmin. Accurate determination of CuEXC may help with response guided medical therapy for WD. In addition, another parameter, relative exchangeable copper (REC), the ratio of CuEXC to total serum copper has been shown to be a highly accurate tool for the diagnosis of WD. REC may also be helpful in distinguishing patients with WD carrying one abnormal $A T P 7 B$ allele. Using a cut-off of $15 \%$, REC determination was able to distinguish patients with WD from individuals carrying one abnormal $A T P 7 B$ allele versus normal subjects. Thus, if validated, CuEXC and REC can potentially be used in screening patients' family members as well as monitoring treatment efficacy $(9,10)$.

Another method of monitoring free copper includes a new method of anion exchange chromatography-triple quadrupole inductively coupled plasma mass spectrometry for simple quantification of ceruloplasmin (23). Previously used immunoassays for ceruloplasmin activity rely on indirect methods and can overestimate the quantity of ceruloplasmin due to presence of the apoprotein. This method of anion exchange chromatography-triple quadrupole inductively coupled plasma mass spectrometry allows for ceruloplasmin quantification with limit of detection of $0.1 \mathrm{mg} \cdot \mathrm{L}^{-1}$ and limit of quantification $\left.0.4 \mathrm{mg} \cdot \mathrm{L}^{-1}\right)$. Measurement of non-ceruloplasmin bound copper was also studied using this device and noted that the ultrafiltration method to quantify non-ceruloplasmin copper (NCC) is subjected to overestimation in measurement due to a removal of copper from ceruloplasmin (23). This assay may provide a more accurate understanding of the NCC in patients with WD.

\section{Conclusions}

Overall, there are multiple diagnostic, treatment and monitoring modalities for patients with WD. Although further studies are ongoing, the technological advances in genetic testing, imaging, and treatment discussed provide optimism for a more precise detection and management of $\mathrm{WD}$ in the coming years.

\section{Acknowledgments}

We would like to acknowledge the Wilson Disease Association for its assistance to patients with Wilson disease and support of research and educational efforts to help those affected by this disorder.

Funding: None.

\section{Footnote}

Provenance and Peer Review: This article was commissioned by the Guest Editors (Luca Fabris and Mario Strazzabosco) for the series "Recent Advances in Rare Liver Diseases" published in Translational Gastroenterology and Hepatology. The article was sent for external peer review organized by the Guest Editors and the editorial office.

Conflicts of Interest: All authors have completed the ICMJE uniform disclosure form (available at http:// dx.doi.org/10.21037/tgh-2020-02). The series "Recent Advances in Rare Liver Diseases" was commissioned by the editorial office without any funding or sponsorship. Dr. UT is a consultant for Univar Solutions and Ultragenyx pharmaceutical. Dr. MLS receives grant support from Alexion and GMPO, outside the submitted work. The authors have no other conflicts of interest to declare.

Ethical Statement: The authors are accountable for all aspects of the work in ensuring that questions related to the accuracy or integrity of any part of the work are appropriately investigated and resolved.

Open Access Statement: This is an Open Access article distributed in accordance with the Creative Commons Attribution-NonCommercial-NoDerivs 4.0 International License (CC BY-NC-ND 4.0), which permits the noncommercial replication and distribution of the article with the strict proviso that no changes or edits are made and the original work is properly cited (including links to both the formal publication through the relevant DOI and the license). See: https://creativecommons.org/licenses/by-nc-nd/4.0/.

\section{References}

1. Wilson SAK. Progressive Lenticular Degeneration: A familial nervous disease associated with cirrhosis of the liver. Brain 1912;34:295-507.

2. Bearn AG. Genetic and biochemical aspects of Wilson's 
disease. Am J Med 1953;15:442-9.

3. Tanzi RE, Petrukhin K, Chernov I, et al. The Wilson disease gene is a copper transporting ATPase with homology to the Menkes disease gene. Nat Genet 1993;5:344-50.

4. Weiss KH, Thurik F, Gotthardt DN, et al. Efficacy and safety of oral chelators in treatment of patients with Wilson disease. Clin Gastroenterol Hepatol 2013;11:102835.e1-2.

5. Broniek-Kowalik K, Dziezyc K, Litwin T, et al. Anterior segment optical coherence tomography (AS-OCT) as a new method of detecting copper deposits forming the Kayser-Fleischer ring in patients with Wilson disease. Acta Ophthalmol 2019;97:e757-e760.

6. Peng F, Lutsenko S, Sun X, et al. Imaging Copper Metabolism Imbalance in Atp7b -/- Knockout Mouse Model of Wilson's Disease with PET-CT and Orally Administered 64CuCl2. Mol Imaging Biol 2012;14:600-7.

7. Alkhalik Basha MA, Refaat R, Ahmed AF, et al. Brain magnetic resonance spectroscopy (MRS) as a diagnostic tool for detecting early neurological changes in children with Wilson's disease. Eur J Radiol 2019;111:41-6.

8. Jung S, Whiteaker JR, Zhao L, et al. Quantification of ATP7B Protein in Dried Blood Spots by Peptide Immuno-SRM as a Potential Screen for Wilson's Disease. J Proteome Res 2017;16:862-71.

9. El Balkhi S, Trocello JM, Poupon J, et al. Relative exchangeable copper: a new highly sensitive and highly specific biomarker for Wilson's disease diagnosis. Clin Chim Acta 2011;412:2254-60.

10. Guillaud O, Brunet AS, Mallet I, et al. Relative exchangeable copper: A valuable tool for the diagnosis of Wilson disease. Liver Int 2018;38:350-7.

11. Fox AN, Schilsky M. Once daily trientine for maintenance therapy of Wilson disease. Am J Gastroenterol 2008;103:494-5.

12. Ala A, Aliu E, Schilsky ML. Prospective pilot study of a single daily dosage of trientine for the treatment of Wilson disease. Dig Dis Sci 2015;60:1433-9.

13. Weiss KH, Askari FK, Czlonkowska A, et al. Bischoline tetrathiomolybdate in patients with Wilson's disease: an open-label, multicentre, phase 2 study. Lancet Gastroenterol Hepatol 2017;2:869-76.

14. Weiss KH, Czlonkowska A, Hedera P, et al. WTX101 - an investigational drug for the treatment of Wilson disease. Expert Opin Investig Drugs 2018;27:561-7.

15. Camarata MA, Ala A, Schilsky ML. Zinc Maintenance Therapy for Wilson Disease: A Comparison Between
Zinc Acetate and Alternative Zinc Preparations. Hepatol Commun 2019;3:1151-8.

16. Lichtmannegger J, Leitzinger C, Wimmer R, et al. Methanobactin reverses acute liver failure in a rat model of Wilson disease. J Clin Invest 2016;126:2721-35.

17. Müller JC, Lichtmannegger J, Zischka H, et al. High spatial resolution LA-ICP-MS demonstrates massive liver copper depletion in Wilson disease rats upon Methanobactin treatment. J Trace Elem Med Biol 2018;49:119-27.

18. Einer C, Leitzinger C, Lichtmannegger J, et al. A HighCalorie Diet Aggravates Mitochondrial Dysfunction and Triggers Severe Liver Damage in Wilson Disease Rats. Cell Mol Gastroenterol Hepatol 2019;7:571-96.

19. Murillo O, Luqui DM, Gazquez C, et al. Long-term metabolic correction of Wilson's disease in a murine model by gene therapy. J Hepatol 2016;64:419-26.

20. Murillo O, Moreno D, Gazquez C, et al. Liver Expression of a MiniATP7B Gene Results in Long-Term Restoration of Copper Homeostasis in a Wilson Disease Model in Mice. Hepatology 2019;70:108-26.

21. Park SM, Vo K, Lallier M, et al. Hepatocyte transplantation in the Long Evans Cinnamon rat model of Wilson's disease. Cell Transplant 2006;15:13-22.

22. Sauer V, Siaj R, Stoppeler S, et al. Repeated transplantation of hepatocytes prevents fulminant hepatitis in a rat model of Wilson's disease. Liver Transpl 2012;18:248-59.

23. Solovyev N, Ala A, Schilsky M, et al. Biomedical copper speciation in relation to Wilson's disease using strong anion exchange chromatography coupled to triple quadrupole inductively coupled plasma mass spectrometry. Anal Chim Acta 2020;1098:27-36.

24. Roberts EA, Schilsky ML. Diagnosis and treatment of Wilson disease: an update. Hepatology 2008;47:2089-111.

25. Cumings JN. The effects of B.A.L. in hepatolenticular degeneration. Brain 1951;74:10-22.

26. Denny-Brown D, Porter H. The effect of BAL (2,3-dimercaptopropanol) on hepatolenticular degeneration (Wilson's disease). N Engl J Med 1951;245:917-25.

27. Walshe JM. Wilson's disease; new oral therapy. Lancet 1956;270:25-6.

28. McQuaid A, Lamand M, Mason J. The interactions of penicillamine with copper in vivo and the effect on hepatic metallothionein levels and copper/zinc distribution: the implications for Wilson's disease and arthritis therapy. J Lab Clin Med 1992;119:744-50.

29. Scheinberg IH, Sternlieb I, Schilsky M, et al. Penicillamine 
may detoxify copper in Wilson's disease. Lancet 1987;2:95.

30. Brewer GJ, Terry CA, Aisen AM, et al. Worsening of neurologic syndrome in patients with Wilson's disease with initial penicillamine therapy. Arch Neurol 1987;44:490-3.

31. Pall HS, Williams AC, Blake DR. Deterioration of Wilson's disease following the start of penicillamine therapy. Arch Neurol 1989;46:359-61.

32. Walshe JM. Treatment of Wilson's disease with trientine (triethylene tetramine) dihydrochloride. Lancet 1982;1:643-7.

33. Jacquelet E, Beretti J, De-Tassigny A, et al. Compliance with treatment in Wilson's disease: On the interest of a multidisciplinary closer follow-up. Rev Med Interne 2018;39:155-60.

34. Brewer GJ, Dick RD, Johnson V, et al. Treatment of Wilson's disease with ammonium tetrathiomolybdate. I. Initial therapy in 17 neurologically affected patients. Arch Neurol 1994;51:545-54.

doi: 10.21037/tgh-2020-02

Cite this article as: Moini M, To U, Schilsky ML. Recent advances in Wilson disease. Transl Gastroenterol Hepatol 2021;6:21.
35. Brewer GJ, Johnson V, Dick RD, et al. Treatment of Wilson disease with ammonium tetrathiomolybdate. II. Initial therapy in 33 neurologically affected patients and follow-up with zinc therapy. Arch Neurol 1996;53:1017-25.

36. Brewer GJ, Hedera P, Kluin KJ, et al. Treatment of Wilson disease with ammonium tetrathiomolybdate: III. Initial therapy in a total of 55 neurologically affected patients and follow-up with zinc therapy. Arch Neurol 2003;60:379-85.

37. Brewer GJ. Tetrathiomolybdate anticopper therapy for Wilson's disease inhibits angiogenesis, fibrosis and inflammation. J Cell Mol Med 2003;7:11-20.

38. Roberts EA. Update on the Diagnosis and Management of Wilson Disease. Curr Gastroenterol Rep 2018;20:56.

39. Merle U, Encke J, Tuma S, et al. Lentiviral gene transfer ameliorates disease progression in Long-Evans cinnamon rats: an animal model for Wilson disease. Scand J Gastroenterol 2006;41:974-82. 